Kyle Leggott, MD; Corey Lyon, DO; Liza Claus, PharmD, BCACP

University of Colorado Department of Family Medicine, Aurora (Dr. Leggott); University of Colorado Family Medicine Residency, Denver (Drs. Lyon and Claus)

EDITOR

Shailendra Prasad, MBBS, MPH

University of Minnesota North Memorial Family Medicine Residency

Program, Minneapolis

doi: 10.12788/jfp.0112

\section{Consider this Rx for patients with high triglycerides?}

\section{PRACTICE CHANGER}

Consider icosapent ethyl, 2 g twice daily, for secondary prevention of adverse cardiovascular events in patients with elevated triglycerides who are already taking a statin.

STRENGTH OF RECOMMENDATION

B: Based on a single, good-quality, multicenter, randomized controlled trial.

Bhatt DL, Steg PG, Miller M, et al; REDUCE-IT Investigators. Cardiovascular risk reduction with icosapent ethyl for hypertriglyceridemia. NEngl J Med. 2019;380:11-22. ${ }^{1}$

\section{ILLUSTRATIVE CASE}

A 63-year-old man with a medical history significant for myocardial infarction (MI) 5 years ago presents to you for an annual exam. His medications include a daily aspirin, angiotensin-converting enzyme inhibitor, beta-blocker, and a high-intensity statin for coronary artery disease (CAD). On his fasting lipid panel, his low-density lipoprotein (LDL) level is $70 \mathrm{mg} / \mathrm{dL}$, but his triglycerides remain elevated at $200 \mathrm{mg} / \mathrm{dL}$ despite dietary changes. In addition to lifestyle modifications, what can be done to reduce his risk of another MI?

$\mathrm{P}$ atients with known cardiovascular disease (CVD) or multiple risk factors for CVD are at high risk of cardiovascular events, even when taking primary or secondary preventive medications such as statins. ${ }^{2,3}$ In these patients, elevated triglycerides are an independent risk factor for increased rates of cardiovascular events. ${ }^{4,5}$

The 2018 American College of Cardiol-
ogy/American Heart Association (ACC/AHA) guidelines for the treatment of blood cholesterol recommend statin therapy for moderate $(175-499 \mathrm{mg} / \mathrm{dL})$ to severe $(\geq 500 \mathrm{mg} / \mathrm{dL})$ hypertriglyceridemia in appropriate patients with atherosclerotic CVD risk $\geq 7.5 \%$, after appropriately addressing secondary causes of hypertriglycidemia. ${ }^{6}$

Previous studies have shown no benefit from combination therapy with triglyceridelowering medications (eg, extended-release niacin and fibrates) and statins, compared with statin monotherapy. ${ }^{7}$ A recent metaanalysis concluded that omega-3 fatty acid supplements offer no reduction in cardiovascular morbidity or mortality, whether taken with or without statins. ${ }^{8}$

Interestingly, the randomized controlled Japan EPA Lipid Intervention Study (JELIS) demonstrated fewer major coronary events in patients with elevated cholesterol, with or without CAD, who took eicosapentaenoic acid (EPA) - a subtype of omega-3 fatty acids-plus a statin, compared with statin monotherapy. ${ }^{9}$

The REDUCE-IT trial evaluated icosapent ethyl, a highly purified EPA that has been shown to reduce triglycerides and, at the time this study was conducted, was approved for use solely for the reduction of triglyceride levels in adults with severe hypertriglyceridemia. ${ }^{10,11}$

\section{STUDY SUMMARY}

\section{Patients with known CVD had fewer} cardiovascular events on icosapent ethyl The multicenter, randomized controlled 
REDUCE-IT trial evaluated the effectiveness of icosapent ethyl, 2 g orally twice daily, on cardiovascular outcomes. ${ }^{1}$ A total of 8179 patients, $\geq 45$ years of age with hypertriglyceridemia and known CVD or $\geq 50$ years with diabetes and at least 1 additional risk factor and no known CVD, were enrolled at 473 participating sites in 11 countries, including the United States.

Patients had a triglyceride level of 150 to $499 \mathrm{mg} / \mathrm{dL}$ and an LDL cholesterol level of 41 to $100 \mathrm{mg} / \mathrm{dL}$, and were taking a stable dose of a statin for at least 4 weeks. The enrollment protocol was amended to increase the lower limit of triglycerides from 150 to $200 \mathrm{mg} /$ $\mathrm{dL}$ about one-third of the way through the study.

Among the study population, $70.7 \%$ of patients were enrolled for secondary prevention (ie, had established CVD) and $29.3 \%$ of patients were enrolled for primary prevention (ie, had diabetes and at least 1 additional risk factor but no known CVD). Exclusion criteria included severe heart failure, active severe liver disease, glycated hemoglobin $>10 \%$, a planned surgical cardiac intervention, history of pancreatitis, or allergies to fish or shellfish products.

IOutcomes. The primary end point was a composite outcome of cardiovascular death, nonfatal MI, nonfatal stroke, coronary revascularization, or unstable angina.

I Results. The median duration of followup was 4.9 years. From baseline to 1 year, the median change in triglycerides was an $18 \%$ reduction in the icosapent ethyl group but a $2 \%$ increase in the placebo group. Fewer patients in the icosapent ethyl group than the placebo group had a composite outcome event ( $17 \%$ vs $22 \%$, respectively; hazard ratio $[\mathrm{HR}]=0.75 ; 95 \%$ confidence interval $[\mathrm{CI}]$, 0.68-0.83; number needed to treat [NNT] to avoid 1 primary end point event $=21$ ). Patients with known CVD had fewer composite outcome events in the icosapent ethyl group than the placebo group ( $19 \%$ vs $26 \%$; $\mathrm{HR}=0.73$; 95\% CI, 0.65-0.81; NNT = 14) but not in the primary prevention group vs the placebo group (12\% vs $14 \%$; HR $=0.88 ; 95 \%$ CI, 0.70-1.1).

In the entire population, all individual outcomes in the composite were significantly fewer in the icosapent ethyl group (cardio- vascular death: $\mathrm{HR}=0.8 ; 95 \% \mathrm{CI}, 0.66-0.98$; fatal or nonfatal MI: $\mathrm{HR}=0.69 ; 95 \% \mathrm{CI}$, 0.58-0.81; revascularization: $\mathrm{HR}=0.65$; 95\% CI, 0.55-0.78; unstable angina: $\mathrm{HR}=0.68$; 95\% CI, 0.53-0.87; and fatal or nonfatal stroke: $\mathrm{HR}=0.72 ; 95 \% \mathrm{CI}, 0.55-0.93)$. All-cause mortality did not differ between groups $(\mathrm{HR}=0.87$; 95\% CI, 0.74-1.02).

No significant differences in adverse events leading to discontinuation of the drug were reported between groups. Atrial fibrillation occurred more frequently in the icosapent ethyl group (5.3\% vs $3.9 \%$ ), but anemia $(4.7 \%$ vs $5.8 \%)$ and gastrointestinal adverse events (33\% vs $35 \%$ ) were less common.

\section{WHAT'S NEW}

\section{First RCT to demonstrate value of pairing icosapent ethyl with a statin}

Many prior studies on use of omega-3 fatty acid supplements to treat hypertriglyceridemia did not show any benefit, possibly due to a low dose or low ratio of EPA in the study drug. ${ }^{8}$ One trial (JELIS) with favorable results was an open-label study, limited to patients in Japan. The REDUCE-IT study was the first randomized, placebo-controlled trial to show that icosapent ethyl treatment for hypertriglyceridemia in patients with known CVD who are taking a statin results in fewer cardiovascular events than statin use alone.

Also worth noting: Since publication of the REDUCE-IT study, the FDA has approved an expanded indication for icosapent ethyl for reduction of risk of cardiovascular events in statin-treated patients with hypertriglyceridemia and established CVD or diabetes and $\geq 2$ additional cardiovascular risk factors. ${ }^{11}$

\section{CAVEATS}

\section{Drug's benefit was not linked to triglyceride level reductions}

The cardiovascular benefits of icosapent ethyl were obtained irrespective of triglyceride levels achieved. This raises the question of other potential mechanisms of action of icosapent ethyl in achieving cardiovascular benefit. However, this should not preclude the use of icosapent ethyl for secondary prevention in appropriate patients.
In this multinational trial of patients with hypertriglyceridemia and CVD or cardiovascular risk factors, icosapent ethyl significantly reduced the risk of cardiovascular events. 
I Laboratory testing is helpful, but not needed. Testing for tinea capitis would require that you obtain a sample from the affected area using a swab, edge of a scalpel blade, or scalp brush. ${ }^{7}$ Because treatment can require weeks of medication, diagnosis should be confirmed with a $\mathrm{KOH}$ or culture when possible.

\section{Newer antifungals \\ provide a Tx advantage}

Oral antifungal medications are the treatment of choice for tinea capitis. Newer antifungals, such as terbinafine and fluconazole, require a 3- to 6-week course compared to the standard 6- to 8-week course of griseofulvin. ${ }^{1}$ Also, antifungal shampoos-such as those that contain selenium sulfide-may be used for topical treatment but only as adjuvant therapy. ${ }^{1,2}$

I For our patient, we dispensed a 3-week course of oral fluconazole, 3 to $6 \mathrm{mg} / \mathrm{kg}$, to be given daily by his parents. We also recommended the use of an antidandruff shampoo, if possible. The treatment outcome was not known because our team's humanitarian global health trip had ended.

JFP

\section{References}

1. Usatine R, Smith MA, Mayeaux Jr EJ, Chumley HS. The Color Atlas and Synopsis of Family Medicine. 3rd ed. New York, NY: McGrawHill; 2019.

2. Handler MZ. Tinea capitis. Medscape. https://emedicine medscape.com/article/1091351-overview. Updated February 21, 2020. Accessed November 30, 2020.

3. Hernández-Bel P, Malvehy J, Crocker A, et al. Comma hairs: a new dermoscopic marker for tinea capitis [in Spanish]. Actas Dermosifiliogr. 2012;103:836-837.

4. Mirmirani P, Lue-Yen T. Epidemiologic trends in pediatric tinea capitis: a population-based study from Kaiser Permanente Northern California. J Am Acad Dermatol. 2013;69:916-921.

5. Mikaeili A, Kavaoussi H, Hashemian AH, et al. Clinico-mycological profile of tinea capitis and its comparative response to griseofulvin versus terbinafine. Curr Med Mycol. 2019;5:15-20.

6. Slowinska M, Rudnicka L, Schwartz RA, et al. Comma hairs: a dermatoscopic marker for tinea capitis: a rapid diagnostic method. Journal of the American Academy of Dermatology. 2008;59(suppl 5):S77-S79.
CONTINUED FROM PAGE 519

\section{CHALLENGES TO IMPLEMENTATION}

\section{Medication is pricey}

Icosapent ethyl is an expensive medication, currently priced at an estimated $\$ 351 /$ month using a nationally available discount pharmacy plan, although additional manufacturer's discounts may apply. ${ }^{12,13}$ The cost of the medication could be a consideration for widespread implementation of this recommendation. JFP

\section{ACKNOWLEDGEMENT}

The PURLs Surveillance System was supported in part by Grant Number UL1RR024999 from the National Center For Research Resources, a Clinical Translational Science Award to the University of Chicago. The content is solely the responsibility of the authors and does not necessarily represent the official views of the National Center For Research Resources or the National Institutes of Health.

Copyright (c) 2020. The Family Physicians Inquiries Network. All rights reserved.

\section{References}

1. Bhatt DL, Steg PG, Miller M, et al; REDUCE-IT Investigators. Cardiovascular risk reduction with icosapent ethyl for hypertriglyceridemia. NEngl J Med. 2019;380:11-22.

2. Bhatt DL, Eagle KA, Ohman EM, et al; REACH Registry Investigators. Comparative determinants of 4 -year cardiovascular event rates in stable outpatients at risk of or with atherothrombosis. JAMA. 2010;304:1350-1357.

3. Cannon $\mathrm{CP}$, Braunwald E, McCabe $\mathrm{CH}$, et al; Pravastatin or Atorvastatin Evaluation and Infection Therapy-Thrombolysis in Myocardial Infarction 22 Investigators. Intensive versus moderate lipid lowering with statins after acute coronary syndromes [published correction appears in NEngl J Med. 2006;354:778]. N EnglJ Med. 2004;350:1495-1504.

4. Klempfner R, Erez A, Sagit BZ, et al. Elevated triglyceride level is independently associated with increased all-cause mortality in patients with established coronary heart disease: twenty-twoyear follow-up of the Bezafibrate Infarction Prevention Study and Registry [published correction appears in Circ Cardiovasc Qual Outcomes. 2016;9:613]. Circ Cardiovasc Qual Outcomes. 2016;9:100-108.

5. Nichols GA, Philip S, Reynolds K, Granowitz CB, Fazio S. Increased cardiovascular risk in hypertriglyceridemic patients with statin-controlled LDL cholesterol. J Clin Endocrinol Metab. 2018;103:3019-3027.

6. Grundy SM, Stone NJ, Bailey AL, et al. 2018 AHA/ACC/AACVPR/ AAPA/ABC/ACPM/ADA/AGS/APhA/ASPC/NLA/PCNA guideline on the management of blood cholesterol: a report of the American College of Cardiology/American Heart Association Task Force on Clinical Practice Guidelines [published correction appears in J Am Coll Cardiol. 2019;73:3237-3241]. J Am Coll Cardiol. 2019;73:e285-e350.

7. Ganda OP, Bhatt DL, Mason RP, Miller M, Boden WE. Unme need for adjunctive dyslipidemia therapy in hypertriglyceridemia management. J Am Coll Cardiol. 2018;72:330-343.

8. Aung T, Halsey J, Kromhout D, et al; Omega-3 Treatment Trialists' Collaboration. Associations of omega-3 fatty acid supplement use with cardiovascular disease risks: meta-analysis of 10 trials involving 77917 individuals. JAMA Cardiol. 2018;3:225-234.

9. Yokoyama M, Origasa H, Matsuzaki M, et al; Japan EPA lipid intervention study (JELIS) Investigators. Effects of eicosapentaenoic acid on major coronary events in hypercholesterolaemic patients (JELIS): a randomised open-label, blinded endpoint analysis [published correction appears in Lancet. 2007;370:220]. Lancet. 2007;369:1090-1098.

10. Ballantyne CM, Bays HE, Kastelein JJ, et al. Efficacy and safety of eicosapentaenoic acid ethyl ester (AMR101) therapy in statintreated patients with persistent high triglycerides (from the ANCHOR study). Am J Cardiol. 2012;110:984-992.

11. FDA approves use of drug to reduce risk of cardiovascular events in certain adult patient groups [news release]. Silver Spring, MD US Food and Drug Administration; December 13, 2019. www. fda.gov/news-events/press-announcements/fda-approves-usedrug-reduce-risk-cardiovascular-events-certain-adult-patientgroups. Accessed November 30, 2020.

12. Vascepa. GoodRx. www.goodrx.com/vascepa. Accessed Novem ber 30, 2020.

13. The VASCEPA Savings Program. www.vascepa.com/getting started/savings-card/. Accessed November 30, 2020 